\title{
CLOCKS, COMPUTERS, BLACK HOLES, SPACETIME FOAM, AND HOLOGRAPHIC PRINCIPLE
}

\author{
Y. JACK NG \\ Department of Physics and Astronomy, University of North Carolina, Chapel Hill, NC 27599-3255, USA \\ E-mail:yjng@physics.unc.edu
}

\begin{abstract}
What do simple clocks, simple computers, black holes, space-time foam, and holographic principle have in common? I will show that the physics behind them is inter-related, linking together our concepts of information, gravity, and quantum uncertainty. Thus, the physics that sets the limits to computation and clock precision also yields Hawking radiation of black holes and the holographic principle. Moreover, the latter two strongly imply that space-time undergoes much larger quantum fluctuations than what the folklore suggests - large enough to be detected with modern gravitational-wave interferometers through future refinements.
\end{abstract}

\section{Introduction \& Summary}

Computers and clocks are physical systems. As such, they must obey the laws of physics. Here we show that, according to the laws of quantum mechanics and gravitation, the number $\nu$ of operations per unit time, and the number $I$ of bits of information in the memory space of a simple computer ("simple" in the sense to be made precise below), are both limited such that their product is bounded by a universal constant given by $I \nu^{2} \lesssim t_{P}^{-2}$, where $t_{P}=\left(\hbar G / c^{5}\right)^{1 / 2} \sim 10^{-43} \mathrm{sec}$ is the Planck time. We also show that the total running time $T$ over which a simple clock can remain accurate, and the smallest time interval $t$ that the clock is capable of resolving, are bounded by $T t^{-3} \lesssim t_{P}^{-2}$. Remarkably, nature appears to make use of such simple clocks and simple computers, for these bounds are saturated for black holes. Then we show that the physics that sets the limits to computation and clock precision is precisely the physics that governs the quantum fluctuations of space-time leading to space-time foam 2.3 . These quantum fluctuations induce an uncertainty $\delta R$ in the measurement of distance $R$ given by $R(\delta R)^{-3} \lesssim l_{P}^{-2}$ (in contrast to $\delta R \gtrsim l_{P}$, independent of $R$, according to the forklore 4 ) where $l_{P}=c t_{P} \sim 10^{-33} \mathrm{~cm}$ is the Planck length. Interestingly, such uncertainties in distance measurements are exactly what are necessary to yield the holographic principle that the number of degrees of freedom that can be put into a region of space is bounded by the area of the region in Planck units. And significantly, these quantum fluctuations of space-time can be detected with modern gravitational-wave interferometers perhaps in the nottoo-distant future.

\section{Two Ingredients}

The ingredients we will use are the general principles of quantum mechanics and general relativity. First let us follow Wigner to use quantum mechanics to set fundamental limits on the mass $m$ of any clock. For the clock to give time to within accuracy $t$ repeatedly throughout its running time $T$, it must have a spread in position $\delta R$ (throughout $T$ ) so small that the time at which a light quantum strikes it (in order to read it) can be determined within accuracy $t: \delta R \lesssim c t$. But if the clock has a linear spread of $\delta R$, then its momentum uncertainty is $\hbar(\delta R)^{-1}$. After a time $\tau$, its position spread grows to $\delta R(\tau)=\delta R+\hbar \tau m^{-1}(\delta R)^{-1}$ with the minimum at $\delta R=(\hbar \tau / m)^{1 / 2}$. At the end of the total running time $T$, the linear spread can grow to

$$
\delta R \gtrsim\left(\frac{\hbar T}{m}\right)^{1 / 2} .
$$

But this spread in position is required to be less that ct. Hence, for a given $T$ and $t$, the bound on $m$ reads

$$
m \gtrsim \frac{\hbar}{c^{2} t}\left(\frac{T}{t}\right) .
$$

This limit is more restrictive than that given by Heisenberg's energy-time uncertainty relation because the requirement that repeated measurement of time not introduce significant inaccuracies over the total running time $T$ imposes a more severe limit on its mass than a single simultaneous measurement of both the energy $m c^{2}$ and the time $t$.

The second ingredient comes from general relativity. Consider a simple clock consisting of two parallel mirrors (each of mass $m / 2$ ) between which bounces a beam of light. On the one hand, for the clock to be able to resolve time interval as small as $t$, the mirrors must be separated by a distance $d$ with $d / c \lesssim t$. On the other hand, $d$ is necessarily larger than the Schwarzschild radius $G m / c^{2}$ of the mirrors so that the clock does not collapse into a black hole. From these two requirements, it follows

$$
t \gtrsim \frac{G m}{c^{3}} \text {. }
$$




\section{Simple Clocks}

For any simple clock with accuracy $t$ and total running time $T$, we can relate these two time scales by substituting Eq. (2) into Eq. (3) to get

$$
T \lesssim t\left(\frac{t}{t_{P}}\right)^{2} .
$$

Thus the more precise a clock is, i.e., the smaller $\mathrm{t}$ is, the shorter it can keep accurate time, i.e., the smaller $\mathrm{T}$ is. But don't let any clock-salesman talk you into buying a relatively inaccurate clock with the hope that it may last longer, for even a femtosecond $\left(10^{-15} \mathrm{sec}\right)$ precision yields the bound $T \lesssim 10^{34}$ years. However, note that the bound on $T$ goes down rapidly as $t^{3}$.

Let us now explain what we mean by the qualification "simple" characterizing the simple clock (and the simple computer). To do that, consider a large clock consisting of $\mathrm{N}$ identical small clocks to keep time one after another. For large enough N, the T-t relation (Eq. (4)) and Eq. (3) are obviously violated for the large clock. But fortunately, this argument is not valid if we consider only those clocks for which no such separation of components is involved. They are what we call simple clocks. The same qualification will be understood to apply to simple computers.

\section{Simple Computers}

Since a computer can serve as a clock, the limit Eq. (4) also applies to a computer. To obtain a universal bound on the speed of computation and the memory space of any simple information processor, we note that the fastest possible processing frequency is given by $\nu=t^{-1}$ and $T / t$, the maximum number of steps of information processing, is, aside from factors like $\ln 2$, the amount of information $I$ that can be registered by the computer. Then it is an easy matter to use the T-t relation in Eq. (4) to show that

$$
I \nu^{2} \lesssim \frac{1}{t_{P}^{2}} \equiv \frac{c^{5}}{\hbar G} \sim 10^{86} / \sec ^{2}
$$

independent of the mass, size, and details of the simple computer. This universal bound (valid for any simple computer) links together our concepts of information, gravity, and quantum uncertainty. We will see below that nature seems to respect this bound which, in particular, is realized for black holes. For comparison, current laptops perform about $10^{10}$ operations per sec on $10^{10} \mathrm{bits}$, yielding $I \nu^{2} \sim 10^{30} / \sec ^{2}$, and the current largest $I \nu^{2}$ is around $10^{39} / \sec ^{2}$ set by the largest IBM SP cluster.

\section{Black Holes}

If a black hole is used as a clock, then it is reasonable to expect that the maximum running time of this gravitational clock and the minimum time interval that it can be used to measure are given by the Hawking black hole life-time and the light travel time across the black hole's horizon respectively:

$$
T \sim \frac{G^{2} m^{3}}{\hbar c^{4}}, \quad t \sim \frac{G m}{c^{3}} .
$$

(Alternatively we can derive the above equation by appealing to Wigner's two inequalities Eq. (1) and Eq. (2) and using the Schwarzschild radius of the black hole as the minimum clock size. 9 Thus, if we had not known of black hole evaporation, this remarkable result would have implied that there is a maximum lifetime for a black hole when quantum observers are introduced.) Now, according to the second half of Eq. (6), the limit on $t$ as shown in Eq. (3) is saturated for a black hole. Furthermore, using both expressions in Eq. (6) one can easily show that the bound given by Eq. (2) is saturated. It then follows that the subsequent bounds (Eq. (14) and Eq. (5)) are also saturated for black holes. Thus, black hole are the ultimate simple clocks and the ultimate simple computers. It is curious that although they can be very massive and large, black holes are basically simple — a fact further supported by the no-hair theorem.

Here let us comment on the main difference between our approach and that of Lloyd 10 on the physical limits to computation. Lloyd's use of Heisenberg's energy-time uncertainty principle to find $\nu$ is tantamount to putting $T \sim t$ in Wigner's inequality (Eq. (2)). Thus, while we have introduced two time scales $T$ and $t$, Lloyd has introduced only $t$. But as the case of black holes shows, these two time scales are not the same in general. For a 1-kg black hole, according to Lloyd $10, \nu \sim 10^{51} / \mathrm{sec}$ and $I \sim 10^{16}$ bits; but according to us, Eq. (2) and Eq. (3) yield $\nu \sim 10^{35} / \mathrm{sec}$ and $I \sim 10^{16}$ bits. Thus we disagree with the limits given by Lloyd for the case of black holes.

\section{Space-time Foam}

To see the connection between the physical limits to computation and clock precison and the physics that governs the quantum fluctuations of space-time (giving rise to space-time foam), let us consider measuring the distance $R$ between two points. We can put a clock at one of the points and a mirror at the other point. By sending a light signal from the clock to the mirror in a timing experiment we can determine the distance. But the quantum uncertainty in the positions of the clock and the mirror introduces an inaccuracy $\delta R$ in the distance 
measurement. The same argument used above to derive the T-t relation now yields a bound for $\delta R$ :

$$
\delta R\left(\frac{\delta R}{l_{P}}\right)^{2} \gtrsim R
$$

in a distance measurement. 2 In a time measurement, an analogous bound is given by Eq. (4) with $T$ play ing the role of the measured time and $t$ the uncertainty. 2 This limitation to space-time measurements can be interpreted as resulting from quantum fluctuations of spacetime itself. In other words, at short distance scales, space-time is foamy. Thus the same physics underlies both the foaminess of space-time and the limits to computation and clock precision. Not surprisingly, these bounds have the same form.

\section{Holographic Principle}

Furthermore, the same physics is behind the holographic principle 5 . To see this, consider a region of space with linear dimension $\mathrm{R}$. The conventional wisdom claims that the region can be partitioned into cubes as small as $\left(l_{P}\right)^{3}$. It follows that the number of degrees of freedom of the region is bounded by $\left(R / l_{P}\right)^{3}$, i.e., the volume of the region in Planck units. But the conventional wisdom is wrong this time, for according to Eq. (7), the smallest cubes into which we can partition the region cannot have a linear dimension smaller than $\left(R l_{P}^{2}\right)^{1 / 3}$. Therefore, the number of degrees of freedom of the region is bounded by $\left[R /\left(R l_{P}^{2}\right)^{1 / 3}\right]^{3}$, i.e., the area of the region in Planck units, as stipulated by the holographic principle.

Two comments are in order: (1) Since black holes have an entropy given by the event horizon in Planck units, 11 the holographic bound is saturated for black holes. We can see this also by using Eq. (6). (2) As argued above, the holographic principle has its origin in quantum fluctuations of space-time. So, turning the argument around, we believe the holographic principle alone suggests that the quantum fluctuations of spacetime are as given by Eq. (7) and hence are much larger than what the conventional wisdont leads us to believe.

\section{Smoking Gun}

Both the Hawking radiation of black holes and the holographic principle lend strong albeit indirect supports for the space-time foam model given by Eq. (7). At present there is no solid direct evidence; after all, even on the size of the whole observable universe ( $\sim 10^{10}$ ligh-years $)$, Eq. (17) yields a fluctuation fo only about $10^{-13} \mathrm{~cm}$. Luckily, as pointed out recently 6 , modern gravitational-wave interferometers, through future refinements, may reach displacement noise level low enough to test this space-time foam model. To see this, in any distance measurement that involves a time interval $\tau$, we note that there is a minute uncertainty $\delta R \sim\left(c \tau l_{P}^{2}\right)^{1 / 3}$. This uncertainty manifests itself as a displacement noise (in addition to other sources of noises) that infests the interferometers. The Fourier transform of this noise gives the displacement amplitude spectral density of frequency $f$ :

$$
S(f) \sim f^{-5 / 6}\left(c l_{P}^{2}\right)^{1 / 3} .
$$

By comparing the spectral density with the existing observed noise level of $3 \times 10^{-17} \mathrm{~cm}-\mathrm{Hz}^{-1 / 2}$ near $450 \mathrm{~Hz}$, the lowest noise level reached by the Caltech 40 -meter interferometer, we obtain the bound $l_{P} \lesssim 10^{-27} \mathrm{~cm}$. The "advanced phase" of LIGO is expected to achieve a noise level low enough to probe $l_{P}$ down to $10^{-31} \mathrm{~cm}$, only about two orders of magnitude from what we expect it to be! (For comparison, if the conventional space-time foam model $\left.⿴ 囗 \delta R \gtrsim l_{P}\right)$ is correct, even the advanced phase of LIGO can probe $l_{P}$ only down to $10^{-17} \mathrm{~cm}$.) Eagerly we wait for our interferometer colleagues to improve the sensitivity of modern gravitational-wave interferometers by another two orders of magnitude to provide us with the "smoking gun" evidence of space-time foam (Eq. (7)). Perhaps then, for the first time, we will catch a glimpse of the very fabric of space-time!

\section{Acknowledgments}

This work was supported in part by the U.S. Department of Energy under \#DE-FG05-85ER-40219.

\section{References}

1. Y. J. Ng, gr-qc/0006105.

2. Y. J. Ng and H. van Dam, Mod. Phys. Lett. A9, 335 (1994); ibid. A10, 2801 (1995).

3. F. Karolyhazy, Il Nuovo Cimento A42, 390 (1966); N. Sasakura, Prog. Theor. Phys. 102, 169 (1999); hep-th/0001161.

4. C.W. Misner, K.S. Thorne, and J.A. Wheeler, Gravitation (Freeman, San Francisco, 1973).

5. G. 't Hooft, in Salamfestschrift, edited by A. Ali et al. (World Scientific, Singapore, 1993), 284; L. Susskind, J. Math. Phys. 36, 6377 (1995).

6. G. Amelino-Camelia, Nature 398, 216 (1999); Phys. Lett. B477, 436 (2000).

7. Y. J. Ng and H. van Dam, Found. Phys. 30, 795 (2000); Phys. Lett. B477, 429 (2000).

8. H. Salecker and E. P. Wigner, Phys. Rev. 109, 571 (1958).

9. J. D. Barrow, Phys. Rev. D10, 6563 (1996).

10. S. Lloyd, Nature 406, 1047 (2000).

11. J.D. Bekenstein, Phys. Rev. D7, 2333 (1973); J.A. Wheeler, Int. J. Theor. Phys. 21, 557 (1982). 